\title{
Glaucoma Detection Using Dwt Based Energy Features and Ann Classifier
}

\author{
Nitha Rajandran \\ Department of Computer Science,Mahatma Gandhi University Caarmel Engineering \\ College,Perunad,Kerala,India
}

\begin{abstract}
Glaucoma is a disease in which fluid pressure in the eye increases continously, and damage the optic nerve and leads to vision loss. It is the second leading cause of blindness. For identification of disease in human eyes we are using clinical decision support system which is based on retinal image analysis technique, that used to extract structure,contextual or texture features. Texture features within images which gives accurate and efficicent glaucoma classification.For finding this texture features we use energy distribution over wavelet subband.In this paper we focus on fourteen features which is obtained from daubecies(db3),symlets(sym3), and biorthogonal(bio3.3,bio3.5, and bio3.7). We propose a novel technique to extract this energy signatures using 2$D$ wavelet transform and passed these signatures to different feature ranking and feature selection strategies. The energy obtained from detailed coefficent are used to classify normal and glaucomatous image with high accuracy. This will be classified using support vector machines, sequential minimal optimization, random forest, naive Bayes and artificial neural network. We observed an accuracy of $94 \%$ using the ANN classifier.Performance graph is shown for all classifiers. Finally the defected region founded by segmentation and this will be post processed by morphological processing technique for smoothing operation.
\end{abstract}

Index Terms: Artificial neural network, data mining,feature extraction, glaucoma,imagetexture, wavelet transforms.

\section{Introduction}

GLAUCOMA is an eye disease and it is the second leading cause of peripheral blindness nowadays. This leads to neurodegeneration of the optic nerve.It is estiamted that above six million peoples have glaucoma[1], and half of them are unaware that they have this.Approximately 140000 are blind from glaucoma,about $5 \%$ of population between $40-50$ yers old and $10 \%$ over 70 years old ,whcih increases the risk of significant vision loss.

Glaucoma is normally associated with increased fluid pressure in the eye.It is divided into two types "open-angle and "closed-angle" glaucoma.Closed -angle glaucoma appears suddenly which is painful and also visual loss occurs quickly.Open-angle is a chronic glaucoma, It progresses at a slower rate and patients may not notice they have lost vision until the disease has increased significantly. In closed angle glaucoma, fluid pressure in the eye increases because of inadequate fluid flow between iris and the cornea.

For the detection and pigmentation of glaucoma,effective quantitative imaging alternatives are offered in biomedical imaging.Eye images manually analysing is fairly time consuming,and the accuracy of parameter measurements varies between experts.Some of the prominent existing modalities and their enhancements,includes optical coherence tomography[2] and multifocal electroretinograph (mfERG)

[3],are teh most prominent techniques employed for the quantitatively analyze stuctural and functional abnormalities in the eye, which will observe variability and to quantify the progresion of the disease.

Automated clinical decision support system(CDSSs) in opthalmology[4], which are designed mainly for the identification of the disease pathology in human eyes.CDSSs are based on the retinal image analysis techniques that are used to extract structural,textural or contextual features from the images and it effectively distinguish between normal and diseased samples.Retinal image analysis technique which is mainly based on computational techniques to make qualitative checking of eye more objectively.Main goal behind this method is to , reduce the variability that may arising between different clinicla trackingsand for the progresion of the structural characteristics of the human eye.

In CDSSs features extracted are categorized into two types structural features and texture features.In structural features commonly includes disk area ,disk diameter ,rim area,cup diameter,cup-to-disk ratio, and topological features extracted from the image[5].Proper orthogonal decomposistion technique (POD) which uses structural features to identify glaucomatous progression[6].Pixel level information is given by POD which is used to gauge significant changes across samples which is locatrion or region specific. The measurement of texture features, is roughly defined as spatial variation of pixel intensity.Texture featrures are not bound to any specific location on image.Several feature extraction techniques are there to mine texture features.From the experiments, we can say that texture based techniques have been proven successful. 
In this paper glaucomatous image is classified using texture features. Wavelet transform(WT) in image processing is used to obtain the texture features.In this WT the content of the image which is represented in frequency domain and it is independent in size. Wavelet Fourier analysis (WFA)can be able to characterize neuroanatomic disruption in glaucoma.

It is a mathematical model to analyze and parameterize the tem-poral, superior nasal, inferior, and temporal (TSNIT) [7] shapesDWT is a multiscale analysis method, in which analysis can be performed on different scales. In this approach, discrete wavelet transform (DWT) uses a fourth-order symlets wavelet which can be able to extract features and analyze discontinuities and abrupt changes contained in signals. At each level, the transformation provides an analysis of source image at a different resolution, which leads to independent approximation and detailed coeffi-cients. In this it applied fast Fourier transform (FFT)[8] for the detailed coefficients. The resultant Fourier amplitudes are combined with the normalized approximation coefficients of the DWT to create a set of features. This will provide valuable information for the analysis of images using mathematical techniques by the DWT and FFT.

In this paper we use image processing for classifying normal and glaucomatous images accurately for that we use the wavelet energy of each wavelet subband by implementing wavelet packet based texture classification [9] for differentiating the potential of the texture features obtained from the image [10]. It is based on the distribution of average texture features obtained from three prominent wavelet families. Hence, our objective is to evaluate and finding out the most important features for glaucomatous image classification.

We examine the effectiveness of different wavelet filters on the basis of glaucomatous images by taking the standard 2-D-DWT. We use three mostly using wavelet filters, the daubechies (db3), the symlets (sym3), and the biorthogonal (bio3.3, bio3.5, and bio3.7) filters [11]. We calculated the averages of the detailed horizontal and vertical coefficients and wavelet energy signature from different energy coefficients. After this energy signatures are subjected to feature ranking and feature selection schemes for determining the best combination of features and aid in the convergence of classifiers, such as the support vector machine (SVM), sequential minimal optimization (SMO), random forest, naive Bayes and artificial neural network techniques. This different classifiers are used to find the normal and glaucomatous image and it find out which classifier can be able to find accurately the glaucomatous image. Artificial neural network is trained and it will classify the fourteen energy signatures and with an accuracy of $94 \%$ it find out infected image. By the pigmentation it also finds out where the glaucoma infected and it will post processed for the morphological processing tehcnique for smoothing operation.

The paper is organized in the following way. Section II contains a brief account of related work. Section III contain explanation of dataset used. Section IV contains detailed description of the proposed syssystem and different steps used for this research. SectionV contains a description of the classifiers and classifier parameters employed for our experiments. Section VI includes experimental results obtained for the proposed method.Finally the paper concludes by Section VII.

\section{Related Work}

Glaucoma is a disease which occurs by the degeneration of optic nerves(optic disc). It is an optic neuropathy that causes the loss of retinal ganglion cells and damage to the retinal nerve fibre layer (RNFL) [12].The thinning of RNFL is a direct indicator of the glaucoma disease.For the early detection of glaucoma objective and quantitative measurements of RNFL is calculated.For this method such as OCT,SLP,mfERG have been used for the thickness and phase retardation respectively.Polarization-sensitive OCT is a combination of OCT and SLP.In human retina it has been examined using time domain PS-OCT and spectral domain.To diagnose and observe glaucoma measurement changes in the retinal nerve fibre layer thicknes should be calculated. Measuring ocular bloodflow is another one diagnostic and a supervision tool for glaucoma.In this literature survey briefly describes some of the techniques that are used for the detection of glaucoma.

In Optical coherence tomography (OCT) uses near-infrared light to provide direct cross-sectional measurement of the RNFL. The principles employed are alike to those used in B-mode ultrasound except light, which have not sound, it is used to create the 2-dimensional images. The light source can be directed into the eye through a conservative slit-lamp biomicroscope and focused on the retina through a distinctive 78diopter lens. This system requires dilation of the patient's pupil. Another one method introduced by Alexandrescus Dascular AM introduced a Confocal scanning laser ophthalmoscopy(CSLO), which is a laser based image gaining which is proposed to improve quality of the examination compared to ordinary ophthalmologic examination. A laser id scanned crossways there along with a detector system. To estimate the width of theRNFL, a single spot on the retina is illuminated at any time,ensure a high-contrast image of great reproducibility that can be used. This technique does not need maximal mydriasis, which may be a problem in patients having glaucoma. The Heidelberg Retinal Tomography is another one technique used in glaucoma detection. 
Another one existing works is SLP which is introduced by Ferreri F, Aragona P. RNFL, it is used to access the RNFL for early detection of glaucoma. This technology have several advantages because less biological variability is expected in the region of the RNFL than the optic nerve head. It is also independent of the reference plane, which causes a change in the state of divergence of a laser beam as it passes. It uses a 780$\mathrm{nm}$ diode to illuminate optic nerve. The polarization state of the light emerging from the eye is evaluated and linked with RNFL thickness. Unlike CSLO, scanning laser polarimetry (SLP) can unswervingly measure the thickness of the RNFL. GDx is an example of a scanning laser polarimeter. GDx contain a normative database and statistical software package for permit comparison to age matched normal subjects of the same racial origin. The advantages of this system ais that images can be obtained without pupil dilation, and evaluation can be done roughly in 10 minutes. Modern instruments have added improved and erratic corneal compensation technology. Multi focal electo retinal graph which proposed by J. M. Miquel-Jimenez[12] proposed the Glaucoma detection by wavelet-based analysis of the global flash multifocal electroretinogram. Existing clinical analysis of the multifocalelectroretinography (mfERG) recording for detecting glaucoma is based on standard signal morphology, which can be measured by the amplitudes and latencies. This analysis is not sensitive enough for detection of minute changes in the multifocal electroretinogram signals. Here another method for detection of open angle glaucoma based on the categorization of global flash mfERG signals isgiven. The digital signal processing technique is based on the wavelets for the detection of advanced-stage glaucoma. Two markers were obtained from the recorded signals by applying the discrete wavelet transform, which help discriminate healthy from glaucomatous signals.

Another one structural features extracted by Pulsatile Ocular Blood Flow. The pulsatile in ocular pressure is due to the flow of blood into the eye during cardiac systole. Pulsatile ocular blood flow can detect by the permanent monitoring of intra ocular pressure. This can be changed into a volume measurement using the identified relationship between ocular pressure and ocular volume. Pulsatile blood flow is principally determined by the choroidal vessels which is mainly applicable to patients with glaucoma, since the optic nerve is supplied in large part by choroidal circulation. The optical coherence tomography and multifocal electro retino graph (mfERG) are well-known methods working in order to analyze functional abnormality of the eye especially glaucoma. Another one important method is Computer Based Diagnosis of Glaucoma using Digital Fundus Images.

Archana Nandibewoor [13] introduced the Computer Based Diagnosis of Glaucoma using Digital Fundus Images. Images having different color variation inside the eye is compared by using images taken by high definition laser camera. These are called as fundus images. MATLAB software tool is used to extract features from these fundus images. We can find out whether a person is suffering from glaucoma by measuring the color pixels at the affected areas. To identify whether the person is suffering from Glaucoma, a test is made using the image of a normal person which is kept as reference (say zero) and then compared with the clinical observations of the person's image. In this section it describes the existing system used in this area.

Ours is bit different from the existing system we used to extract the texture features and its extracted using energy signatures. This signatures are passed for the feature selection and feature ranking process. After that it is accurately classifying whether it is normal or glaucomatous image. Finally we experimentally prove that better classifier is Artificial neural network with an accuracy of $94 \%$. By the pigmentation it also finds out where the glaucoma infected and it will post processed for the morphological processing technique for smoothing operation.

\section{Dataset}

The retinal images taken for this research were collected from Vasan eye care hospital, Kerala, India.The doctors in the department of opthalmology,based on the quality and usability of samples they manually curated. The committee consisit of senior doctors, approved the use of image for this studies. All images are taken with aresolution of $560 \times 720$ pixels and -

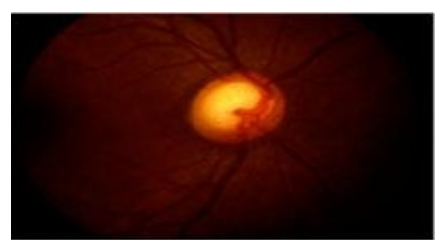

(a)

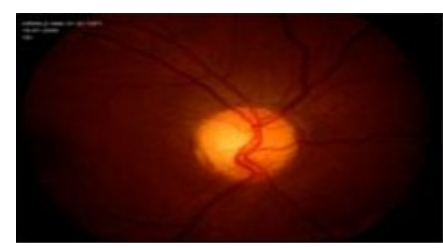

(b)

Fig. 1. Fundus images (a) normal (b) glaucoam.In glaucoma, the pressuers within the eye's vitreous chamber increases and compromises the blood vessels of the optic nerve head,leading to permanent loss of axonsof the vital ganglion cells 
they are stored in lossless JPEG format[10].In this dtaset it contain 60 fundus images: 30 normal and 30 open angle glaucomatous images from 20 to 70 years old.For diagnosing this disease,a fundus camer,microscope and a light source used.Fig. 1(a) and (b) presents normal and glaucoma fundus images respectively.

\section{Proposed System}

In this paper proposing a system which is for effectively detecting the glaucomatous eye. Because in now a days glaucoma is the second leading cause of blindness. In most of the case problem get serious because of undetection in later stages from the normal images. In this paper research is made for an early detection of glaucoma wavelet based energy features. For this dataset images were subjected to histogram equalization. The main objective for applying histogram equalization [14] depends on two things: to assign the intensity values of pixels in the input images, which the ouptut image contained the unfiorm distribution of the intensities. This input image are then passed for feature extraction and feature ranking procedures.

\section{A. Discrete Wavelet Transform-Based Features}

The DWT captures both spatial and frequency informa-tions of a signal. It analyzes the images by decomposing it into a coarse approximation via low-pass filtering and into detail information via high-pass filtering. By this decomposition it performed recursively on low-pass approximation coefficients obtained at each level, until when the necessary iterations are reached.

Let each image be represented as a p x q gray-scale matrix $I[i, j]$, where each element in the matrix represents the gray-scale intensity of one pixel of that image. Each nonborder pixel which have eight adjacent neighboring pixel intensities. These eight neighbors can be used for the traverse in matrix. The resultant 2-DDWT coefficients which is the same irrespective of whether that matrix is traversed right-to-left or left-to-right. Hence, it is sufficient that when we consider four decomposition directions corresponding to $0^{\circ}$ (horizontal, Dh), $45^{\circ}$ (diagonal, Dd), $90^{\circ}$ (vertical, Dv), and $135^{\circ}$ (diagonal, Dd) orientations. The decomposition structure for the one level which is illustrated in Fig. 2. In this figure, I is the image, $g[n]$ and $h[n]$ which is the low-pass and high-pass filters, respectively, and A is the approximation coefficient. In this study, the results from level 1 it is found to yield significant features.

From the Fig. 2 it is clearly noted that, the first level of decomposition results in four coefficient matrices, whcih is named as, A1, Dh1, Dv1, and Dd1. Since the number of elements

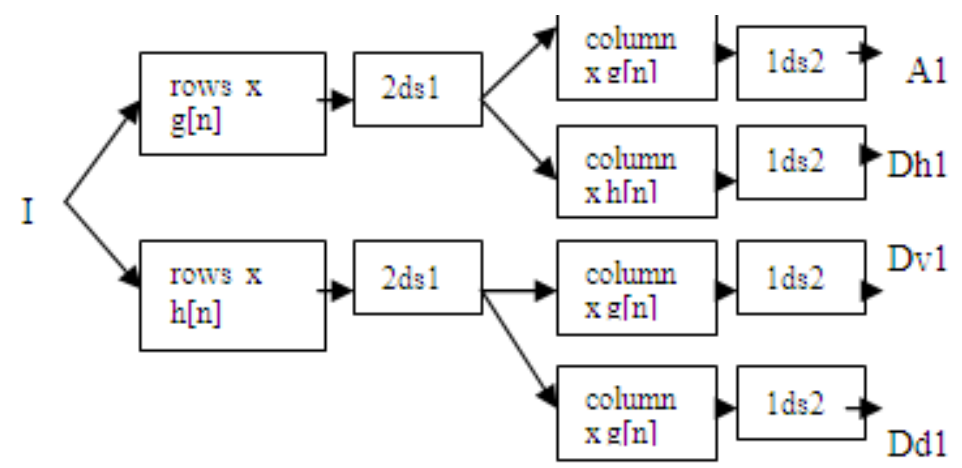

Fig.2. 2-D-DWT decomposition:2ds1 indicates whcih rows are down sampled by two and column by one.1ds2 indicates that the rows are down sampled by one and column by two.The "x" operator indicates convolution

operation

Dd1.Since the number of elements in this matrices are very high. The three features are defined by the DWT coefficents.Equation (1) and (2) whcih determine the average of the intensity values, whereas (3) is an average of the energy in intensiy values.

$$
\begin{aligned}
& \text { - Average Dv1 }=\underset{\text { p x q }}{1} \sum_{\mathrm{x}=\{\mathrm{p}\}} \sum_{\mathrm{y}=\{\mathrm{q}\}}|\operatorname{Dh} 1(\mathrm{x}, \mathrm{y})| \\
& \text { Average Dh1 } \left.=\underset{\mathrm{pxq}}{1} \sum_{\mathrm{x}=\{\mathrm{p}\}} \sum_{\mathrm{y}=\{\mathrm{Dv}\}} \mid \mathrm{x}, \mathrm{y}\right) \mid \\
& \text { Energy }=\underset{p x q}{1} \sum_{x=\{p\}} \sum_{y=\{q\}}(\operatorname{Dv} 1(x, y))^{2}
\end{aligned}
$$




\section{B. Preprocessing of Features}

Fourteen features can be found from the normal and glaucomatous samples.Their corresponding distribution across this samples.From this fourteen features $\mathrm{p}$ value exhibiting $<0.0001$ were choosen for the analysis.

\section{Normalization of Features}

This fourteen features are subjected for z-score normalization.In this z-score a sample consist of 14 features which is converted for zero mean and unit variance. The mean and standard deviation of the input vector are computed as follows.

$$
\mathrm{y}_{\text {new }}=\underset{\substack{\text { old } \\ \text { std }}}{\mathrm{y}_{\text {s. }}}
$$

\begin{tabular}{|c|c|c|c|}
\hline Features & Normal & Glaucoma & P-VALUE \\
\hline $\begin{array}{l}\text { db3: } \\
\text { Dh1_Average_II } \\
\text { Norm }\end{array}$ & $1.07 \pm 0.273$ & $0.757 \pm 0.108$ & $<0.0001$ \\
\hline$\overline{\mathrm{d}} \mathrm{b} 3: \mathrm{cV}$ _Energy & $\begin{array}{l}3.801 \mathrm{E}- \\
06 \pm 4.253 \mathrm{E}- \\
06\end{array}$ & $2.528 \mathrm{E}-05 \pm 1.023 \mathrm{E}-05$ & $<0.0001$ \\
\hline $\begin{array}{l}\text { Sym3:Dh1_Aver } \\
\text { age_II_Norm }\end{array}$ & $1.07 \pm 0.273$ & $0.757 \pm 0.108$ & $<0.0001$ \\
\hline 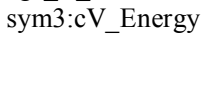 & $\begin{array}{l}3.801 \mathrm{E}- \\
06 \pm 4.253 \mathrm{E}- \\
06\end{array}$ & $2.528 \mathrm{E}-05 \pm 1.023 \mathrm{E}-05$ & $<0.0001$ \\
\hline $\begin{array}{l}\text { Rbio3.3: } \\
\text { Dh1_Average_II } \\
\text { Norm }\end{array}$ & $1.77 \pm 0.511$ & $1.34 \pm 0.154$ & $<0.0001$ \\
\hline $\begin{array}{l}\text { rbio3.3:cD_Ener } \\
\text { gy }\end{array}$ & $\begin{array}{l}1.413 \mathrm{E}- \\
04 \pm 3.504 \mathrm{E}- \\
04\end{array}$ & $6.066 \mathrm{E}-04 \pm 2.498 \mathrm{E}-04$ & $<0.0001$ \\
\hline $\begin{array}{l}\text { rbio3.3:cV_Ener } \\
\text { gy }\end{array}$ & $\begin{array}{l}3.605 \mathrm{E}- \\
05 \pm 4.214 \mathrm{E}- \\
05\end{array}$ & $1.741 \mathrm{E}-04 \pm 6.184 \mathrm{E}-05$ & $<0.0001$ \\
\hline $\begin{array}{l}\text { Rbio3.5: } \\
\text { Dh1_Average_II } \\
\text { _Norm }\end{array}$ & $1.51 \pm 0.330$ & $1.17 \pm 0.158$ & $<0.0001$ \\
\hline $\begin{array}{l}\text { rbio3.5:cD_Ener } \\
\text { gy }\end{array}$ & $\begin{array}{l}1.240 \mathrm{E}- \\
04 \pm 2.492 \mathrm{E}- \\
04\end{array}$ & $5.517 \mathrm{E}-04 \pm 2.312 \mathrm{E}-04$ & $<0.0001$ \\
\hline $\begin{array}{l}\text { rbio3.5:cv_Energ } \\
\mathrm{y}\end{array}$ & $\begin{array}{l}2.715 \mathrm{E}- \\
05 \pm 3.187 \mathrm{E}- \\
05\end{array}$ & $1.287 \mathrm{E}-04 \pm 4.599 \mathrm{E}-05$ & $<0.0001$ \\
\hline $\begin{array}{l}\text { Rbio3.7: } \\
\text { Dh1_Average_II } \\
\text { Norm }\end{array}$ & $1.36 \pm 0.241$ & $1.09 \pm 0.162$ & $<0.0001$ \\
\hline $\begin{array}{l}\text { rbio3.7:cH_Ener } \\
\text { gy }\end{array}$ & $\begin{array}{l}7.641 \mathrm{E}- \\
05 \pm 5.939 \mathrm{E}- \\
05\end{array}$ & $1.505 \mathrm{E}-04 \pm 5.607 \mathrm{E}-05$ & $<0.0001$ \\
\hline $\begin{array}{l}\text { rbio3.7:cD_Ener } \\
\text { gy }\end{array}$ & $\begin{array}{l}1.474 \mathrm{E}- \\
04 \pm 2.029 \mathrm{E}- \\
04\end{array}$ & $5.246 \mathrm{E}-04 \pm 2.217 \mathrm{E}-04$ & $<0.0001$ \\
\hline $\begin{array}{l}\text { rbio3.7:cV_Ener } \\
\text { gy }\end{array}$ & $\begin{array}{l}2.380 \mathrm{E}- \\
05 \pm 2.776 \mathrm{E}- \\
05\end{array}$ & $1.144 \mathrm{E}-04 \pm 4.148 \mathrm{E}-05$ & $<0.0001$ \\
\hline
\end{tabular}

Table I Wavelet Features For Normal And Glaucomatous Images

\section{Feature Ranking}

This is a preprocessing step which preceedes to classification. We are focuussing on filter based approach to rank the features based on the potential across samples.In this the wavelet features, our analysis consists of four widely used feature ranking schemes. These include chi squared $\left(\left[\chi^{2}\right]\right)[18]$, gain ratio [19], information gain [20] feature evaluation tech-niques, and relief feature ranking schemes [21] that are based on an extensive ranking algorithm.

\section{E. Feature Selection}

For selecting a subset of features,we should make the given set of features for the consistencey subset evaluvation strategy.CSE finds the feature combination and have the values to divide the data into subset 
containing a strong single class majority that is high class consistencey.This consistency measure was first presented in the following way.

Consistency $=1-\quad \frac{\sum_{\mathrm{i}=0}^{\mathrm{j}}\left|\mathrm{D}_{\mathrm{i}}\right|-\left|\mathrm{M}_{\mathrm{i}}\right|}{\mathrm{N}}$

where $s$ if feature subset,$J$ is teh number of distinct combination of features $\left|\mathrm{D}_{\mathrm{i}}\right|$ number of occurences and $\left|\mathrm{M}_{\mathrm{i}}\right|$ is caridnality of majority class.

The various features subset selection schemes followed in this paper are part of the WEKA. suite and it include the best first, random search, genetic search, and greedy step-wise approaches.

Best First: The best first search strategy implementation is based on the beam search algorithm. The rudiments of this algorithm are based on this standard hill climbing approach with backtracking to determine the best fit of a subset of features.

Random Search: In the random search approach, an exhaus-tive list of this random feature combinations are generated and tested. The subset of features that generates the best accuracy which is chosen as the subset of features that best represents the input set.

Genetic Search: The genetic search technique was proposed by, and utilizes neural network feature ranking. This al-gorithm entails the several iterations of the evaluation of a feature subset and includes training a neural network and computing its cost and accuracy.

Greedy Stepwise: Greedy stepwise subset evaluation is per-formed by using the greedy forward or backward search through the feature space.

\section{Classifier Settings}

For performing the validation of ranked features and fea-ture subsets using the standard C-SVC implementation of SVM, SMO, random forest, and naive Bayes (as shown in Table II) [26]-[28]. The SVM employs the radial basis and this function with a predetermined value of gamma set at 0.28 . John C. Platt's SMO algorithm is used to train the SVM [27]. The implementation of SMO is performed by using both the polynomial kernel with the exponential set to 2.5 , and the Pearson VII function-based universal kernel, each having the parameters omega and sigma, which are both set at 0.1 . The number of trees in this random forest algorithm is set at 10 . The nave Bayes classifier is set to use a kernal function to the estimate distribution of the data, rather than assuming that their data follow a normal distribution. The classifier settings are determined based on repeated trials on the training

\section{A. Artificial Neural Network}

Back propagation learning algorithm can considered as the corner stone of ANN. ANN models have been studied and applied in recent years in reaching human performance in different fields.ANNs are emperical model in natrure, they obtain accurate and robust solution for more or less precisely problems and for phenomena understood through experimental data.

Table II Overall Accuracies Of Individual Wavelet Features Using Accuracy Test

\begin{tabular}{lllllll}
\hline \hline Features & $\begin{array}{c}\text { LibSVM } \\
(1)\end{array}$ & SMO_(1) & SMO_(2) & $\begin{array}{c}\text { Random } \\
\text { Forest }\end{array}$ & $\begin{array}{c}\text { Naïve } \\
\text { Bayes }\end{array}$ & ANN \\
\hline db3 & 90.00 & 88.67 & 93.33 & 85.50 & 93.33 & 94.33 \\
Sym3 & 88.33 & 87.33 & 93.33 & 86.00 & 88.17 & 95.17 \\
rbio3.3 & 88.33 & 85.67 & 91.83 & 89.00 & 90.17 & 92.18 \\
rbio3.5 & 88.33 & 88.33 & 91.00 & 90.17 & 90.50 & 94.54 \\
rbio3.7 & 90.00 & 88.67 & 93.33 & 85.50 & 93.33 & 95.34
\end{tabular}

Multilayer perception network is mathematical term with each node i called neuron.Each junction between two neuron $\mathrm{i}$ and $\mathrm{k}$ called synaps or link,A real threshold called activation threshold is associated with neuron i.A transfer functionm determine activation degree of neuron as a function of its threshold,weights of the input and output junction of neuron connected to input synapsis.

Fundamental characteristics of Multilayer percepton network are is an adaptive method which permits carrying on non linear staatistics and fitting ios made by gradient method using taining data.

ANN workflow based on 7 steps commonly 
- collect data

- create network

- configure network

- initialize weight and biases

- train the network

- validate the network

- use the network

\section{Back Propagation Algorithm}

It is an algorithm for adjusting the correct weights between the nodes whcih uses graident decent method to minimise the mean quadratic error between the actual output of the perceptron and the desired output.High performance is achieved with this back propagation algorithm.when compared to gradient method backpropagation is bbetter. A system of AI employing with ANN and integrate the analysis of the fibre retina is used for the diagnosis of the glaucoma.

\section{Feed Forward Network}

It is an ANN network where connection between the unit do not form a directed cycle. The feedforward neural network was the first and simplest type of artificial neural network devised. In this network, the information moves in only one direction, forward, from the input nodes, through the hidden nodes (if any) and to the output nodes. There are no cycles or loops in the network.

This class of networks consists of multiple layers of computational units, usually interconnected in a feed-forward way. Each neuron in one layer has directed connections to the neurons of the subsequent layer. In many applications the units of these networks apply a sigmoid function as an activation function.

\section{Experimental Results}

In this section it provides a detailed description of the results from feature ranking and selection strategies.

\section{Classification}

Energy signatures are subjected to different feature ranking and feature selection strategy.For this we use tenfold cross vaidation and 60:40 slip tests.Both tests which are carried out on the entire 60 samle data set.This data set is splitted into ten parts.In each iteration nine parts are used for training and remaining part for testing. Thus the results obtained from the tenth iteration gets the accurate result.

It is evident that the highest accuracy obtained is $93.33 \%$ using the tenfold cross-validation method. In the case of 60:40 split up, all classifiers except the random forest classifier which presented as the highest accuracy of $95.83 \%$. However, it is possible to obtain the highest accuracy which is using fewer features in CSE shows that the SMO_(2) classifier gives 93.33\% accuracy using just two features can be selected by the best first and greedy stepwise strategies in the CSE method. These features are db3-Dh1_Average_11_Norm and rbio3.3-cD_Ener. Therefore, this features can be considered that highly discriminatory. The consistency of results can be indicates that these features can be perform consistently independent to the classifier. We can conclude that the CSE feature selection method does help in obtaining the highest accuracy using this fewer features, thereby simplifying the implementation of this technique.

Finally, we comparing this results with those obtained using HOS and texture features [10]. The training samples and the test sample identifiers are mapped with those used in and it will enable a direct comparision of accuracy. To establish a baseline analysis of the features used in this study, we gauge this reliability of individual wavelet features by performing this standard sensitivity and specificity analysis on the independent training and test sets used. The classifiers can be used for this analysis are described. We carried out a tenfold cross validation of independent in wavelet features on the entire set, consisting of 60 samples. It can be seen that the SMO_(2) classifier performs consistently well using any wavelet-based feature. From all the given smaples we can prove that the artificial neural network gives the high accuracy classification result.

When the optic nerve is damaged by glaucoma, most of the individual fibers in the nerve are lost, and the optic nerve be-comes excavated. As the glaucoma progresses, more optic nerve tissue is lost and the optic cup grows. These changes make this fundus images obtained from glaucoma patients different from those obtained from normal subjects. We believe that this features selected and evaluated in this study adequately capture these subtle differences in the fundus images and, thus, help the classifiers to detect normal and glaucoma cases with high accuracy. 


\section{Conclusion}

In this paper a new approach for glaucomatous image classification is illustrated.The dependence between the features extracted using three wavelet filters that have been subjected to various feature ranking and feature selection methods. The ranked subsets of selected features have been move to a set of classification algorithms to gauge the effectiveness of these features. From these accuracies obtained and contrasted, we can conclude that the energy obtained from the detailed coefficients can be used to distinguish glaucomatous and normal eye image. The calculations shows that artificial neural network will give more accurate results than other classifier.Performance of each classifiers also shown.Segmentation is done for region detection.

\section{Reference}

[1]. R. Varma et al., "Disease progression and the need for neuroprotection in glaucoma management," Am. J. Manage Care, vol. 14, pp. S15-S19, 2008.

[2]. R. George, R. S. Ve, and L. Vijaya, "Glaucoma in India: Estimated burden of disease,” J. Glaucoma, vol. 19, pp. 391-397, Aug. 2010.

[3]. K. R. Sung et al., "Imaging of the retinal nerve fiber layer with spectral domain optical coherence tomography for glaucoma diagnosis," Br. J. Ophthalmol., 2010.

[4]. J. M. Miquel-Jimenez et al., "Glaucoma detection by wavelet-based anal-ysis of the global flash multifocal electroretinogram," Med. Eng. Phys., vol. 32, pp. 617-622, 2010.

[5]. B. Brown, "Structural and functional imaging of the retina: New ways to diagnose and assess retinal disease," Clin. Exp. Optometry, vol. 91, pp. 504-514, 2008

[6]. S. Weiss, C. A. Kulikowski, and A. Safir, "Glaucoma consultation by computer," Comp. Biol. Med., vol. 8, pp. 24-40, 1978.

[7]. S. Weiss et al., "A model-based method for computer-aided medical decision-making,” Artif. Intell., vol. 11, pp. 145-172, 1978.

[8]. R. O. Duncan et al., "Retinotopic organization of primary visual cortex in glaucoma: A method for comparing cortical function with damage to the optic disk," Invest. Ophthalmol. Vis. Sci., vol. 48, pp. 733-744, Feb. 2007.

[9]. M. Balasubramanian et al., "Clinical evaluation of the proper orthagonal decomposition framework for detecting glaucomatous changes in human subjects," Invest. Ophthalmol. Vis. Sci., vol. 51, pp. 264-271, 2010.

[10]. U. R. Acharya, S. Dua, X. Du, V. S. Sree, and C. K. Chua, "Automated diagnosis of glaucoma using texture and higher order spectra features,” IEEE Trans. Inf. Technol. Biomed., vol. 15, no. 3, pp. 449-455, May 2011.

[11]. S. Dua, U. R. Acharya, and E. Y. K. Ng, Computational Analysis of the Human Eye With Applications. World Scientific Press, 2011.

[12]. E. A. Essock, Y. Zheng, and P. Gunvant, "Analysis of GDx-VCC polarime-try data by wavelet-Fourier analysis across glaucoma stages," Invest. Ophthalmol. Vis. Sci., vol. 46, pp. 2838-2847, Aug. 2005.

[13]. K. Huang and S. Aviyente, "Wavelet feature selection for image classifi-cation," IEEE Trans. Image Process., vol. 17, no. 9, pp. 1709-1720, Sep. 2008.

[14]. A. Arivazhagan and L. Ganesan, "Texture classification using wavelet transform,” Pattern Recog. Lett., vol. 24, pp. 1513-1521, 2003.

[15]. I. Daubechies, Ten Lectures on Wavelets. Philadelphia, PA: Society for Industrial and Applied Mathematics, 1992.

[16]. R. C. Gonzalez and R. E. Woods, Digital Image Processing. NJ: Prentice Hall, 2001.

[17]. M. H. Dunham, Data Mining Introductory and Advance Topics. NJ: Pren-tice Hall, 2002.

[18]. H. Liu and R. Setiono, "Chi2: Feature selection and discretization of numeric attributes," in Proc. IEEE 7th Int. Conf. Tools With Artif. Intell., 1995, pp. 338-391.

[19]. J. R. Quinlan, "Induction of desion trees," Mach. Learning, vol. 1, pp. 81- 106, 1986.

[20]. J. R. Quinlan, C4.5 Programs for Machine Learning. San Mateo: Morgan Kaufmann, 1993.

[21]. K. Kira and L. A. Rendell, “A practical approach to feature selection,” in Proc. 9th Int. Workshop Mach. Learning, San Francisco, CA, 1992, PP.249-256. 